\title{
ETA-INVARIANTS AND VON NEUMANN ALGEBRAS
}

\author{
R. G. DOUGLAS, S. HURDER AND J. KAMINKER
}

1. Main theorem. Let $M$ be a compact oriented Riemannian manifold of dimension $4 k-1$. The operator $D={ }^{*} d+d^{*}$ acting on the $2 k-1$ forms on $M$ is selfadjoint, and for any Hermitian vector bundle $E \rightarrow M$ with connection $\nabla$, there is a selfadjoint operator $D \otimes \nabla$ acting on the smooth sections of $\Lambda^{2 k-1}\left(T^{*} M\right) \otimes E$. The data $(D, E)$ defines a class $[D, E]$ in the odd analytic $K$-homology group $K_{1}^{a n}(M)$. We develop in this work an equality between two methods of pairing $[D, E]$ with real-valued $K^{1}$ cohomology classes.

Let $\Gamma=\pi_{1}(M)$ and $A: \Gamma \rightarrow U_{N}$ be a representation, which determines a flat principal $U_{N}$-bundle $P_{A} \rightarrow M$ and an associated flat $C^{N}$-vector bundle $E_{A} \rightarrow M$. If there exists a bundle trivialization $\theta: P_{A} \cong M \times U_{A}$, then it is well known that the pair $(A, \theta)$ represents a class $\bar{A}$ in $K^{1}(M) \otimes R$, $(\S 5$, [APS 2]). The first pairing of $[D, E]$ and $\bar{A}$ uses the relative etainvariant to define the flat bundle index, as in $\left(\S 5\right.$, ibid). Let $\nabla^{0}$ be the flat connection on $M \times U_{N}$ associated to the product structure, and let $\nabla^{1}$ be the flat connection associated with the push-forward under $\theta$ of the flat connection on $P_{A}$. Define a smooth, one-parameter family of selfadjoint operators on smooth sections of $\Lambda^{2 k-1}\left(T^{*} M\right) \otimes E \otimes C^{N}$ by $D_{t}=t \cdot D \otimes$ $\nabla \otimes \nabla^{1}+(1-t) \cdot D \otimes \nabla \otimes \nabla^{0}$. The eta-invariant $\eta\left(D_{t}\right)$ is smooth as a function of $t$ except for a finite number of bounded jump discontinuities, so there exists a well-defined continuous derivative function $\eta\left(D_{t}\right)^{\prime}$ [APS 1,2]. Define

$$
\langle[D, E], \bar{A}\rangle_{\eta}=\int_{0}^{1} \eta\left(D_{t}\right)^{\prime} d t
$$

The second pairing uses the von Neumann algebra $W^{*}(\Gamma)$ associated to the universal covering $\tilde{M}$ of $M$ [A 1]. The lift to $\tilde{M}, \widetilde{D \otimes \nabla}$, of the operator $D \otimes \nabla$ is essentially selfadjoint, and we introduce the projection, $P^{+}$, from $H=L^{2}\left(\tilde{M}, \Lambda^{2 k-1}\left(T^{*} M\right) \otimes E \otimes C^{N}\right)$ onto the positive space $H^{+}$ of $D \otimes \nabla$. The operator $P^{+}$is $\Gamma$-invariant, so defines an element $\left[P^{+}\right]$in $K_{1}\left(W^{*}(\Gamma)\right)$. The data $(A, \theta)$ defines a map $u: \tilde{M} \rightarrow U_{N}$ by restricting the composition $p_{2} \circ \theta: P_{A} \rightarrow M \times U_{N} \rightarrow U_{N}$ to the leaf of $F_{A}$ on $P_{A}$ through a basepoint, where $F_{A}$ is the $U_{N}$-invariant foliation of $P_{A}$ associated to the flat structure. We pair $\left[P^{+}\right]$to $[u] \in K_{(\infty)}^{1}(M)$ by constructing an associated Toeplitz operator, which is Fredholm in the sense of Breuer, and has a von Neumann index which is the continuous dimension of the

Received by the editors August 16, 1988.

1980 Mathematics Subject Classification (1985 Revision). Primary 58G10, 46L80, 57R30.

The first and third author was supported by the NSF.

The second author was supported by the NSF and the Sloan Foundation. 
spectral flow between $P^{+}$and the conjugate $u^{-1} \cdot P^{+} \cdot u$. More precisely, multiplication by $u$ on the coefficients $C^{N}$ defines a bounded operator $M_{u}$ on $H$, and the compression

$$
T(u)=P^{+} \circ M_{u}: H^{+} \rightarrow H^{+}
$$

is $\Gamma$-Fredholm and $\Gamma$-invariant, so as in [A 1] has a $\Gamma$-index. Define

$$
\langle[D, E], \bar{A}\rangle_{\Gamma}=\operatorname{Ind}_{\Gamma}(T(u)) \text {. }
$$

The $\Gamma$-index for a fixed flat bundle $P_{A}$ has an alternate description in terms of the type II von Neumann algebra $W^{*}\left(F_{A}\right)$ associated to the foliation $F_{A}$ by Connes [C 1], with trace derived from the Haar measure on $U_{N}$. The operator $T(u)$ is one of a $U_{N}$-parametrized family of leafwise Toeplitz operators along $F_{A}$, giving index data which is analogous to the hull-completion formulation of the index of almost periodic operators [CDSS].

Our main result is

THEOREM.

$$
\langle[D, E], \bar{A}\rangle_{\eta}=\langle[D, E], \bar{A}\rangle_{\Gamma} .
$$

Moreover, both sides of (3) are equal to a renormalized discrete spectral flow for the lift of $D \otimes \nabla$ to a nonelliptic, pure-point-spectrum $U_{N}$-invariant operator on $P_{A}$.

The theorem is true also for any "geometric operator" on $M$ which is obtained by coupling the Spinor Dirac operator on $M$ to a coefficient vector bundle $E$, so that the theorem applies equally well, for example, to Spin manifolds of odd dimension.

The possibility of relating the eta-invariant for a family $D_{t}$ to a von Neumann dimension as given by (3) above was suggested in (Remark 4, p. 89, [APS 2]).

2. Method of proof. There are three key points to the proof.

(2.1) The invariant $\int_{0}^{1} \eta\left(D_{t}\right)^{\prime} d t$ has a multiplicative property: For $E_{2} \rightarrow$ $M$ a flat vector bundle with fiber dimension $q$ and flat connection $\nabla^{2}$,

$$
\int_{0}^{1} \eta\left(D_{t} \otimes \nabla^{2}\right)^{\prime} d t=q \cdot \int_{0}^{1} \eta\left(D_{t}\right)^{\prime} d t .
$$

We take for $E_{2}$ the infinite-dimensional flat bundle associated to the composition of $A$ with the left regular representation of $U_{N}$ on $L^{2}\left(U_{N}\right)$. The Peter-Weyl Theorem decomposes this infinite bundle into an infinite direct sum of finite-dimensional bundles associated to the characters of irreducible representations of $U_{N}$. The equality (4) is interpreted in a renormalized sense by using the heat kernel, $H_{S}$, on $U_{N}$. We define a central eta-distribution on $U_{N}$ by associating to a class function the weighted sum of the eta-invariants for the operators on $M$ obtained by coupling $D \otimes \nabla$ to the finite-dimensional flat bundles associated to the characteristic subspaces of $L^{2}\left(U_{N}\right)$ in the Peter-Weyl decomposition. Then (4) becomes

$$
\int_{0}^{1} \eta\left(D_{t} ; H_{s}\right)^{\prime} d t=H_{s}(e) \cdot \int_{0}^{1} \eta\left(D_{t}\right)^{\prime} d t .
$$


(2.2) The left-hand side of (5) can be written as the sum of two terms: a spectral flow summand, plus the difference of two values of the etadistribution, one associated to $(D \otimes \nabla) \circ H_{s}$, and the other to $(D \otimes \nabla) \circ$ $\left(u^{-1} H_{s} u\right)$ where now $u$ is the operator on $L^{2}\left(U_{N}\right) \otimes C^{N}$ given by multiplication $u f(g)=g \cdot f(g)$ on the coefficients. Theorem 0.1 of [CG] implies that after renormalizing, the values of the eta-distribution on these two asymptotic functions agree, so that

$$
\lim _{s \rightarrow 0} H_{s}(e)^{-1} \int_{0}^{1} \eta\left(D_{t} ; H_{s}\right)^{\prime} d t=\operatorname{Ind}_{\mathrm{II}}([D, E], \bar{A})
$$

where $\operatorname{Ind}_{\mathrm{II}}([D, E], \bar{A})$ is the renormalized spectral flow of the etadistribution for the family $\hat{D}_{t}$ of operators obtained by lifting $D_{t} \otimes \nabla$ to $P_{A}$ via the leaves of $F_{A}$ which cover $M$. This renormalized spectral flow is also the index, in a suitably renormalized sense, for a Toeplitz operator associated to the p.p.s. operator $\hat{D}_{0}$ and the multiplier $M_{u}$. We call this a renormalized transverse index, as it is based on the construction of the odd $K_{1}$-class for a transversally elliptic operator for the group $U_{N}$-action on $P_{A}$, in analogy with the even transversal index theory of [A 2, S 1, 2].

(2.3) The third point of the proof is to use a Fubini principle, applied to the trace of kernels for operators on $P_{A}$, to show that the renormalized index $\operatorname{Ind}_{\mathrm{II}}([D, E], \bar{A})$ is equal to the index of the leafwise Toeplitz operator on $F_{A}$ described in $\S 1$. The Weyl asymptotic theorem provides the final step, establishing that the renormalized trace converges to the foliation algebra trace formed from Haar measure on $U_{N}$.

3. Final remarks. The main theorem is part of the authors' study of analytic invariants associated to selfadjoint operators on a manifold which are regularized by a foliation on the manifold. This includes both leafwise and transversally elliptic operators for foliations, and we conclude with a discussion of some aspects of this program.

The details of the proof of the main theorem are given in [DHK 3], where we also define for much more general classes of operators than $D=$ ${ }^{*} d+d^{*}$, two types of cyclic cocycles, based on viewing the operator $\widetilde{D \otimes \nabla}$ as either leafwise for $F_{A}$, or transverse for the action of $U_{N}$. A topological index theorem can be derived for the first, while renormalization of the second expresses the relative eta-invariant for $D={ }^{*} d+d^{*}$. A Fubini principle for transverse foliations establishes equality of the first cocycle with the renormalization of the second, and as a corollary yields the index theorem for flat vector bundles of [APS 2]. This procedure can be applied more generally to yield topological formulas for higher order regularized spectral invariants (cf. [D]).

The interpretation of the main theorem applied to $U_{N}=U_{1}$ via quasiperiodic functions was given in [DHK 2], where the main theorem was compared to the results of [CDSS] and a proof was given via Fourier analysis on $U_{1}$.

The index theorem for leafwise selfadjoint elliptic operators can be interpreted via Toeplitz extensions of the foliation $C^{*}$-algebra by the algebra 
of functions on the manifold. In [DHK 4] we identify the analytic index class as an extension in the spirit of the Brown-Douglas-Fillmore theory.

The odd index theorem for coverings is discussed in [H 1], where we give an alternate proof of the main theorem in terms of the eta-invariant for coverings based on [CG]. The spectral properties of leafwise elliptic operators is studied in [ $\begin{array}{ll}\mathbf{H} & 2]\end{array}$; it is expected that the spectral flow interpretation of the eta will extend to all leafwise eta-invariants.

A preliminary report on this work appeared in [DHK 1], where the main theorem was announced with the additional hypothesis that the fundamental group of $M$ be amenable. The outline of our program and the result for the amenable case was discussed in a plenary address at the conference Operator Algebras and Geometry at the Mathematical Sciences Research Institute, Berkeley, June 1985.

\section{REFERENCES}

[A 1] M. F. Atiyah, Elliptic operators, discrete groups and von Neumann algebras, Astérisque 32-33 (1976), 43-72.

[A 2] - Transversally elliptic operators and compact groups, Lecture Notes in Math., vol. 401, Springer-Verlag, Berlin and New York, 1974.

[APS 1] M. F. Atiyah, V. K. Patodi and I. M. Singer, Spectral asymmetry and Riemannian geometry. I, Math. Proc. Cambridge Philos. Soc. 77 (1976), 43-69.

[APS 2] _- Spectral asymmetry and Riemannian geometry. III, Math. Proc. Cambridge Philos. Soc. 79 (1976), 71-99.

[AS] M. F. Atiyah and I. M. Singer, Index theory for skew-adjoint Fredholm operators, Publ. Math. Inst. Hautes Études Sci. 37 (1969), 305-326.

[CG] J. Cheeger and M. Gromov, On the characteristic numbers of complete manifolds of bounded curvature and finite volume, Differential Geometry and Complex Analysis, dedicated to H. E. Rauch (I. Chavel and H. M. Farkas, eds.), Springer-Verlag, Berlin and New York, 1985.

[CD] L. Coburn and R. G. Douglas, On $C^{*}$-algebras of operators on a half-space. I: Index theory, Publ. Math. Inst. Hautes Études Sci. 40 (1971), 59-67.

[CDSS] L. Coburn, R. G. Douglas, R. G. Schaeffer, and I. M. Singer, $C^{*}$-algebras of operators on a half-space. II: Index theory, Publ. Math. Inst. Hautes Études Sci. 40 (1971), 69-79.

[C 1] A. Connes, The von Neumann algebra of a foliation, Lecture Notes in Physics, vol. 80, Springer-Verlag, Berlin and New York, 1978, pp. 145-151.

[C 2] _ Non-commutative differential geometry, I.-The Chern character in K-homology, Publ. Math. Inst. Hautes Études Sci. 62 (1986), 257-309.

[D] R. G. Douglas, Elliptic invariants for differential operators, Proc. Weyl Symposium, Durham, N.C., 1987.

[DHK 1] R. G. Douglas, S. Hurder and J. Kaminker, The eta-invariant, foliation algebras and cyclic cocycles, Math. Sci. Res. Inst., Berkeley, preprint, 1985.

[DHK 2] _ Toeplitz operators and the eta-invariant: the case of $S^{1}$, Contemporary Math., vol. 19, Amer. Math. Soc., Providence, R.I., 1988, pp. 11-41.

[DHK 3] _ Cyclic cocycles, renormalization and eta-invariants, Univ. of Ill., Preprint, 1988.

[DHK 4] _ , The longitudinal cocycle and the index of Toeplitz operators, I.U.-P.U.@I. Preprint, 1988. 1988.

[H 1] S. Hurder, Eta-invariants and the index theorem for coverings, Univ. of Ill., Preprint,

[H 2] _ Analysis and geometry of foliations, Research Monograph, Univ. of Colorado prepublication.

[S 1] I. M. Singer, Some remarks on operator theory and index theory, Lecture Notes in Math., vol. 575, Springer-Verlag, Berlin and New York, 1977, pp. 128-138. 
[S 2] _ Recent applications of index theory for elliptic operators, Proc. Sympos. Pure Math., vol. 23, Amer. Math. Soc., Providence, R.I., 1971, pp. 11-31.

Department of Mathematics, State University of New York, Stony Brook, New YORK 11794

Department of Mathematics, University of Illinois at Chicago, Chicago, ILLINOIS 60680

Department of Mathematics, Indiana University-Purdue University at INDIANAPOLIS, INDIANAPOLIS, INDIANA 46202 
